\title{
A Capacitive Micromachined Ultrasonic Transducer Array for Minimally Invasive Medical Diagnosis
}

\author{
Jingkuang Chen, Xiaoyang Cheng, Chien-Chang Chen, Pai-Chi Li, Senior Member, IEEE, \\ Jian-Hung Liu, and Yu-Ting Cheng
}

\begin{abstract}
A capacitive micromachined ultrasonic transducer (CMUT) array for minimally invasive medical diagnosis has been developed. Unlike traditional ultrasonic transducers, which generally use a bulky piece of substrate, this transducer array was integrated on a 40- $\mu \mathrm{m}$-thick micromachined silicon substrate into a probe shape with a typical shank width of 50-80 $\mu \mathrm{m}$ and a shank length of 4-8 mm. For 1-D arrays, 24-96 CMUT devices were integrated on one such silicon probe and formed an accurately configured phase array. In addition to miniaturization, reduction of the substrate thickness also decreases the intertransducer crosstalk due to substrate Lamb waves. Due to its miniature size, this array can be placed or implanted close to the target tissue/organ and can perform high-resolution high-precision diagnosis and stimulation using high-frequency ultrasounds. The issue of conflict between resolution and penetration depth of ultrasonic diagnosis can therefore be resolved. A two-layer polysilicon surface micromachining process was used to fabricate this device. Suspended polysilicon membranes of diameters ranging from 20 to $90 \mu \mathrm{ms}$ and thicknesses from 1.0 to $2.5 \mu \mathrm{m}$ were used to generate and detect ultrasounds of frequencies ranging from 1 to 10 MHz. B-mode imaging using this transducer array has been demonstrated.

[2007-0125]
\end{abstract}

Index Terms-Acoustic devices, acoustic transducers, actuators.

\section{INTRODUCTION}

$\mathbf{U}$ LTRASOUND has been widely used for medical diagnosis [1] and stimulation [2] for more than 40 years. In an ultrasonic diagnostic process, low-power acoustic waves are used to interact with tissue or organs, and the reflected and transiting ultrasounds can reveal information about anatomical structures, tissue characteristics, blood flow velocity, and muscle movement/contraction in real time. For example, by measuring the propagation speed of ultrasounds in tissue, the tissue temperature can be determined [3]. Piezoelectric thin films [4], [5] have been the most widely used materials for fabricating medical ultrasound transducers. Piezoelectric ultrasonic transducers have been arranged in 1- or 2-D arrays [4], [5], and they are used in different areas of medical diagnosis and stimulation, including destroying cancerous tumors. As a mature technology

Manuscript received August 2, 2006; revised October 5, 2007. Subject Editor K. Najafi.

J. Chen and X. Cheng are with the Department of Electrical and Computer Engineering, The University of New Mexico, Albuquerque, NM 87131 USA (e-mail: jingkuang@yahoo.com).

C.-C. Chen and Y.-T. Cheng are with the Department of Electronics Engineering, National Chiao Tung University, Hsinchu 300, Taiwan, R.O.C.

P.-C. Li and J.-H. Liu are with the Department of Electrical Engineering, National Taiwan University, Taipei 106, Taiwan, R.O.C.

Color versions of one or more of the figures in this paper are available online at http://ieeexplore.ieee.org.

Digital Object Identifier 10.1109/JMEMS.2008.922076 dominating the market, piezoelectric transducers have been designed to transduce ultrasounds of frequencies from kilohertz to up to tens of megahertz, and they are used for both invasive [6] and noninvasive [7] applications. Despite their popularity, piezoelectric transducers have several constraints, which limit their performance in medical diagnosis. Piezoelectric ceramics have relatively poor acoustic impedance match [5] to fluids, particularly gases. For example, the acoustic impedances of lead zirconate titanate (PZT), water, and air are 30 Mrayl $\left(10^{6} \mathrm{~kg} / \mathrm{m}^{2} \cdot \mathrm{s}\right), 1.5 \mathrm{Mrayl}$, and 400 rayl $\left(\mathrm{kg} / \mathrm{m}^{2} \cdot \mathrm{s}\right)$, respectively. With such a large mismatch in acoustic impedance, a large percentage of ultrasounds emitted from a piezoelectric transducer will bounce back at the transducer-fluid interface instead of reaching the target. Although designs including adding impedance matching layers on the transducer have been introduced to alleviate the problem, poor coupling efficiency is still a fundamental limit of piezoelectric ultrasonic systems used in fluids. Additionally, piezoelectric materials are not compatible with monolithic integration of silicon-based electronics. With the increasing number of transducers in today's advanced diagnostic tools and the demand for a better signal-to-noise ratio, difficulty in integrating front-end electronic becomes one of the key factors limiting the performance of piezoelectric ultrasound systems. Due to the fabrication process used to deposit the piezoceramic film, it is difficult to monolithically integrate piezoelectric transducers of different resonant frequencies on one chip. As a result, most piezoelectric ultrasonic tools work at one frequency. In some medical diagnosis, e.g., intravascular ultrasonic imaging, the capability of the tool would be greatly enhanced if transducers of different operating frequencies can be integrated on one head.

Unlike the piezoelectric transducers, which use the expansion and contraction of piezoelectric films to generate and sense ultrasounds, capacitive micromachined ultrasonic transducers (CMUTs) [8]-[10] use the deformation of a suspended thinfilm membrane to transduce ultrasounds. Since 1989, there have been many efforts in applying micromachining technology in capacitive ultrasonic transducer [8]-[10] implementations. A capacitive ultrasonic transducer is composed of a suspended membrane and a counter electrode fixed to the substrate. When an ac signal is applied between the membrane and the fixed electrode, the alternating Coulomb forces drive the membrane to vibrate and generate ultrasonic waves. At the same time, upon reception of impinging ultrasound waves, the membrane deforms and results in changes in the capacitance. This capacitance change is then converted into electrical signals, which reveal the amplitude, frequency, and phase of the ultrasound. 


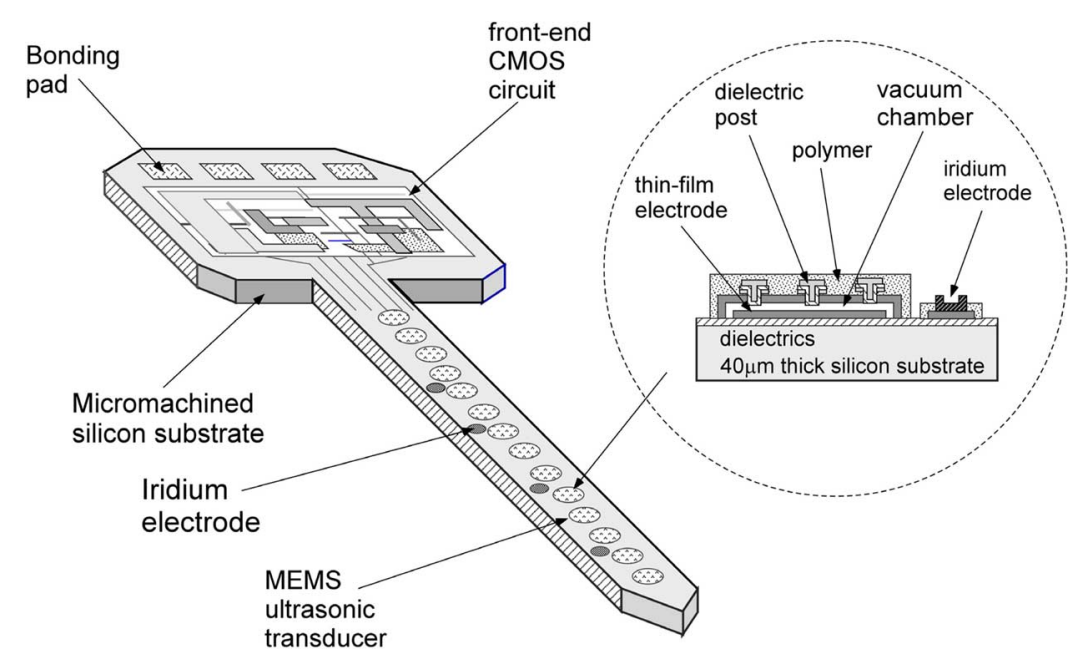

Fig. 1. CMUT array for minimally invasive medical diagnosis. The silicon substrate of the array is micromachined into a probe shape with a cross-sectional dimension as small as $40 \mu \mathrm{m}$ (thickness) $\times 80 \mu \mathrm{m}$ (width) such that the array can be implanted into tissue or inside an organ with a minimal tissue disruption. An array of iridium electrodes is also integrated along the probe shank for electrical stimulation or recording.

Fabricated with the technology originally developed for manufacturing integrated circuits, these micromachined ultrasonic elements can be made into 1- and 2-D arrays [8]-[10] that are compatible with the integration of front-end electronics. Different from piezoelectric ultrasonic devices, which require a film up to more than a millimeter thick for low-frequency operation, the micromachined ultrasonic devices are made of thin films which are typically of a thickness ranging from submicrometer to a few micrometers.

Whether they are piezoelectric or CMUT, one fundamental limit associated with these conventional ultrasonic devices is that they are bulky and restricted by resolution-penetration depth conflict when operated outside the body. Because of this limit, long-wavelength ultrasonic waves are required for detection and/or stimulation of organs and tissues inside the body. For diagnosis or imaging application, long-wavelength acoustic waves limit the detection accuracy and image resolution [11]. For therapeutic stimulation, long-wavelength ultrasounds generally impact an area larger than necessary and could impair the neighboring healthy tissue. Short-wavelength waves are capable of providing more accurate diagnostic information. They are also easier to focus on a small area of the target tissue for stimulation applications. However, they cannot penetrate through the body to reach targets deep inside the tissue. This paper reports the development of a miniaturized CMUT array that is smaller than the human hair and can be placed or implanted into tissue or inside fine vessels for medical diagnosis, stimulation, and/or imaging. Since it can be placed close to the target structure/object, this device can use highfrequency acoustic waves to interact with the target structure and is not constrained by the shallow penetration depth of the short-wavelength waves. This device is useful for small-area high-precision ultrasonic diagnosis/stimulation and near-field high-resolution ultrasonic imaging. It can also be used to measure the blood flow rate of fine vessels or tissue temperature. In stimulation applications, through the use of high-frequency waves, the ultrasounds can be better focused on the target, and the disturbance to neighboring healthy tissue can be minimized.
On this miniature tool, thin-film drum-structured ultrasonic transducers are integrated on a micromachined silicon substrate which has a typical cross-sectional dimension of $40 \mu \mathrm{m}$ (thickness) $\times 80 \mu \mathrm{m}$ (width). The length of this probeshaped tool typically ranges from 2 to $8 \mathrm{~mm}$, depending on how many ultrasound transducer elements are to be integrated on the array. Any 1- or 2-D array can be integrated on this probe substrate. By integrating an array of drum membranes of different geometrical dimensions, a broad frequency spectrum can be generated and detected from the devices on the same substrate built by the same fabrication process. Membrane of different dimensions can be simultaneously used or just switched for operation between two or more frequency bands.

To avoid the process compatibility and the stress problem associated with boron doping, instead of using the dissolving wafer process [12], we have developed a double-sided dryetching micromachining process on a regular silicon wafer to form microprobes as the substrate for the ultrasonic transducer array. Unlike silicon-on-insulator (SOI) micromachined probes [13], this silicon probe uses regular silicon wafers as the substrate and has a cost advantage. Similar to the SOI silicon probes, the substrate dimension of this device can be controlled to within $\pm 2 \mu \mathrm{m}$.

\section{Ultrasonic Transducer Design}

Fig. 1 shows the perspective view of this miniature ultrasound transducer array. In addition to ultrasound transducers, an array of iridium electrode [14] is also integrated on the same substrate such that the device is capable of performing electrical stimulation and recording to/from cells in addition to ultrasound stimulation/sensing. Iridium electrode was chosen for this device because of its stability in tissue fluids and capability of delivering high current density [14]. As shown in the inset of Fig. 1, drum structures made of doped polysilicon are used to transmit and receive ultrasounds. The space between the suspended membrane and the counter electrode is vacuumsealed such that the tissue fluids will not flow into this region 
during device operation. In order to minimize the parasitic capacitance and reduce the $R C$ delay, the width of metal electrical interconnects is reduced to the minimum allowed with the fabrication process, which is $2 \mu \mathrm{m}$ for this prototype run. In order to minimize the parasitic capacitance of the transducer element and the electric interconnect, the thickness of the floor dielectric layer has been increased to $2.1 \mu \mathrm{m}$. The reduction of parasitic capacitance is critical for improving the sensitivity of this device as an acoustic sensor.

In designing this capacitive ultrasonic transducer, five specification parameters are used to determine the performance of the transducer. These parameters are the resonant frequency of the suspended membrane, the maximum membrane displacement (which is specified by the application), the pull down voltage, the bias voltage, and the maximum pressure of the acoustic wave. The pull down voltage is the minimum voltage which will pull the membrane down to touch with the counter electrode. These five parameters determine the membrane thickness, the diameter, and the gap height. For a simple circular membrane with a clamped boundary, the resonant frequency can be determined by the following analytical equation [15]:

$$
\omega_{\mathrm{ns}}=\frac{\lambda_{\mathrm{ns}}}{R^{2}} \sqrt{\frac{K}{\mu}}
$$

where $R$ is the radius of the membrane of uniform thickness, $\lambda_{\mathrm{ns}}$ is a numerical value for circular membrane, $\mu=\rho t$ is the mass of the plate per unit area ( $\rho$ is the plate material density, and $t$ is the plate thickness), and $K=E t^{3} / 12\left(1-\nu^{2}\right)$ is the bending stiffness ( $E$ is the Young's modulus of the membrane material, and $\nu$ is the Poisson's ratio). However, since this transducer is for the operation in tissue fluids, the influence of damping needs to be included, and a numerical method was used to find out the resonant frequency. The center deflection of a clamped circular plate under a uniform pressure can be found from the following equation [15]:

$$
P=\frac{E h^{4}}{R^{4}}\left[\frac{16 y}{3\left(1-\nu^{2}\right) h}+\frac{(7-\nu) y^{3}}{3\left(1-\nu^{2}\right) h^{3}}+\frac{4 R^{2} \sigma y}{(1-\nu) E h^{3}}\right]
$$

where $P$ is the uniform pressure applied on the membrane, $y$ is the center deflection, and $\sigma$ is the intrinsic stress of the membrane material. This equation can be used to estimate the pressure of the ultrasound waves under a prescribed membrane deflection. In this design, the vertical membrane displacement required for the ultrasonic diagnosis or imaging is on the order of several hundred angstroms. In order to minimize the driving voltage and improve the sensitivity of the ultrasound sensor, the gap height between the membrane and the counter electrode was made as small as possible. The membrane thickness and diameter are then determined by the acoustic wave pressure requirement and the resonant frequency. With all these parameters decided, the pull down voltage and the bias voltage can be determined. All these parameters and the performance of the transducers were analyzed by using Coventor's Simulator3D. Fig. 2 shows simulation results of static displacement of the center of a circular 30- $\mu$ m-diameter membrane under different bias voltages on the membrane. Table I lists the mechanical

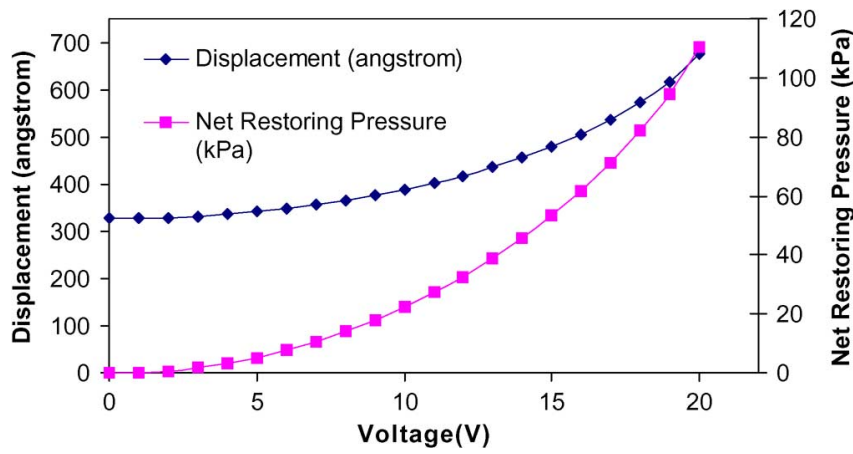

Fig. 2. Simulation result about membrane center displacement and restoring pressure of a $30-\mu$ m-diameter membrane under different bias voltages. The zero-bias displacement is due to pressure difference under and above the membrane. This bilayer membrane is composed of $1.1-\mu$ m-thick parylene $\mathrm{C}$ on top of a $1.5-\mu \mathrm{m}$-thick polysilicon. The resonant frequency of this device is 7.8 $\mathrm{MHz}$.

TABLE I

Properties of THE MATERIALS USED IN THE BiLAYERED CMUT MEMbrane Simulation

\begin{tabular}{|c|c|c|}
\hline properties & polysilicon & Parylene C \\
\hline Young's modulus $E(\mathrm{Gpa})$ & 169 & 4 \\
\hline Poisson ratio $v$ & 0.3 & 0.4 \\
\hline Density $\left(\mathrm{kg} / \mathrm{m}^{3}\right) \rho$ & 2329 & 1289 \\
\hline
\end{tabular}

properties of materials used in this bilayer CMUT simulation. The initial gap height under the membrane is $900 \AA$. This bilayered membrane is composed of a $1.5-\mu$ m-thick polysilicon film and a 1.1- $\mu \mathrm{m}$-thick parylene $\mathrm{C}$ film on top as the passivation layer. The resonant frequency of this membrane is $7.8 \mathrm{MHz}$. Due to the initial pressure difference (which is assumed to be 1 atm) across the membrane, the center of the membrane deforms $327 \AA$ before the application of any electric voltage. Fig. 2 also shows the restoring pressure of the membrane at different deformations.

In order to avoid direct exposure of the tissue/cells to high electrical fields, the suspended membrane is connected to the electrical ground, whereas the counter electrode is connected to the electrical signal line. As the membrane is made of highly conductive material, the shielding effect will constrain the electric field in the region under the membrane. In order to insulate the membrane from the tissue fluids and to protect it from tissue-fluid corrosion, a thin layer of plasma-enhanced chemical vapor deposition (PECVD) oxide and a layer of polymer, which is typically silicone or parylene $\mathrm{C}$, are coated on the surface of the polysilicon membrane as the passivation layers. Polysilicon drums of different diameters, which are ranging from 20 to $90 \mu \mathrm{m}$, have been fabricated for use in the different frequency ranges of interest. Polysilicons of four different thicknesses $(1.0,1.5,2.0$, and $2.5 \mu \mathrm{m})$ were used to construct the membrane. After the final annealing process at $1100{ }^{\circ} \mathrm{C}$ for $60-90 \mathrm{~min}$, the residual stress of these polysilicon films ranged between -5 and $-20 \mathrm{MPa}$.

\section{ISOLATING THIN-FILM CONDUCTORS FROM SILICON NITRIDE}

Conventional polysilicon surface micromachining process uses silicon nitride film as the floor layer to isolate the 


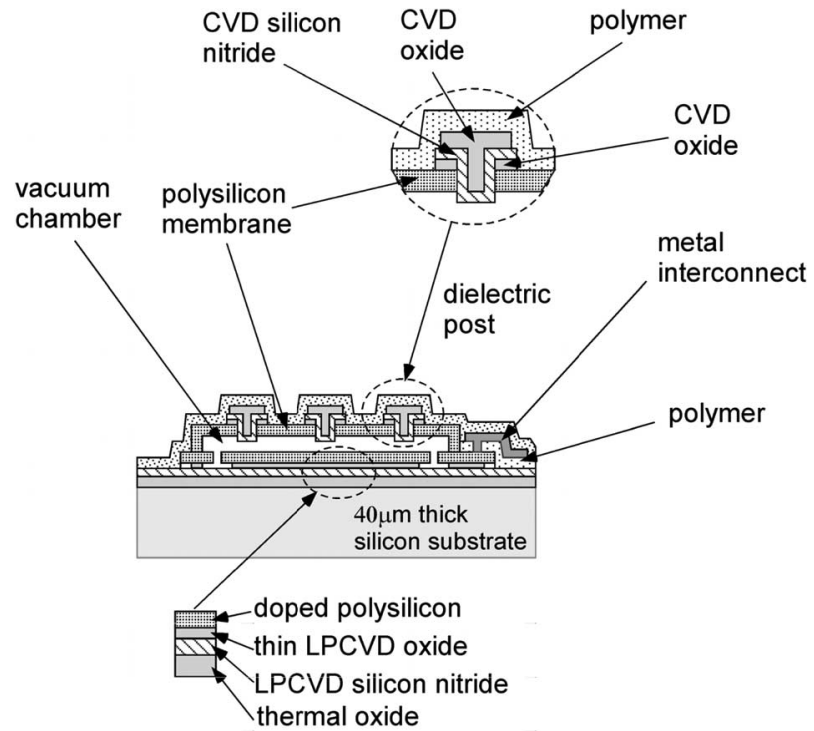

Fig. 3. Cross section of the CMUT device integrated on the silicon probe. The POLY0 structures are designed to anchor on a thin layer of LPCVD oxide to avoid direct contact with the silicon nitride layer. A 2-D array of dielectric post, which protrudes $250-400 \AA$ from the lower surface of the polysilicon membrane, is embedded in the membrane to prevent shorting of the membrane to its counter electrode.

polysilicon microstructures from the substrate. Silicon nitride was chosen mainly because of its low etch rate in hydrofluoric acid (HF) solution (used in the microstructure release process) and its high dielectric strength to sustain a high voltage. While silicon nitride film has reliable mechanical properties and is compatible with MEMS/IC fabrication, its high interface-trap density makes it a poor choice for electrostatic devices. The traps at the nitride-conductor interface capture a significant amount of charge every time a current flows through the conductor. With these charges trapped at the interface, an increasing higher voltage is needed to create a required voltage difference between two pieces of conducting microstructures. This charge-building process continues until the operation voltage is too high to be sustained by the system, and the device will breakdown. This charging process causes a serious reliability problem to many polysilicon electrostatic microdevices.

For a MEMS process using silicon dioxide as the sacrificial layer, silicon nitride is a very convenient material for the floor isolation layer. However, the charging problem limits its capability for use in electrostatic transducers. In order to overcome this dilemma, silicon nitride has to be separated from the conductors so that the charges flowing through the conductor have fewer chances to be trapped at the silicon nitride interface. We have developed a modified polysilicon surface micromachining process for minimizing the nitride-conductor contact area. In order to separate floor silicon nitride film from the first polysilicon layer (POLY0), a thin layer of high-quality low-pressure CVD (LPCVD) silicon dioxide, which is typically 200-250 ̊ thick, is added between the POLY0 electrodes and the floor silicon nitride layer, as shown in Fig. 3. During the subsequent sacrificial release etching, this buffered oxide layer will be undercut by HF. Due to the very thin oxide used in this structure and the etch rate difference between the high-quality LPCVD oxide and the sacrificial phosphosilicate glass (PSG), the undercut is limited to a tolerable range and does not degrade the anchoring strength of POLY0 structures. At the same time, the design rule of minimal POLY0 linewidth has been enlarged to include the impact of lateral etch of buffered thin oxide. In this design, all the microstructures made of the second polysilicon layer (POLY1) anchor to POLY0. As a result, none of the polysilicon structure is in direct contact with the floor nitride. Similarly, a thin layer of polymer, which is typically parylene $\mathrm{C}$, is added between the metal layer and the floor nitride, as shown in Fig. 3, such that metal interconnects do not contact directly to the nitride film.

In order to prevent shorting of the transducer membrane to its counter electrode, a 2-D array of dielectric posts are embedded in the polysilicon membrane, as shown in Fig. 3. These dielectric posts protrude downward about 250-300 ̊ from the lower surface of the polysilicon membrane such that when the membrane is pulled down, they touch the counter electrode first, preventing shorting of the conducting membrane. As shown in the inset of Fig. 3, these dielectric posts are coated with a thin layer of silicon nitride such that they can survive from the HF attack in the membrane release etching process. The typical crosssectional area of these posts is $2 \mu \mathrm{m} \times 2 \mu \mathrm{m}$. In order to minimize the contact areas between the polysilicon membrane and the silicon nitride posts, an LPCVD oxide layer is added on top of the POLY1 layer below the nitride film; thus, the silicon nitride is in direct contact with the POLY1 membrane on the vertical sidewall surfaces of the posts. With this design, the charging problem associated with silicon nitride film can be minimized.

\section{Micromachining the Silicon Substrate}

Traditionally, the most popular approaches for micromachining the silicon substrate to form miniature implantable devices are the dissolving wafer process using boron etch stop [12] and the dry-etching micromachining on an SOI [13] wafer. The dissolving wafer process is able to accurately control the silicon substrate dimension within $\pm 2 \mu \mathrm{m}$, and it has been used to fabricate electrode arrays [12] and multichannel drug delivery chips [16]. The drawback of the boron-defined dissolving wafer process comes from the diffusion process required for doping the silicon and the high tensile stress in the boron-doped silicon. The high-temperature boron diffusion process is not very compatible with transistor and/or some MEMS processes. Additionally, the boron-caused high stress tends to warp the device structure. The SOI micromachining [13] uses doublesided dry etching to form a probe-shaped silicon substrate on the silicon layer of an SOI. The device thickness is determined by the thickness of the SOI structure. The thick substrate under the buried oxide is removed by a backside deep silicon etching. While this approach has a good control on the final device dimension and does not have the stress problem, it requires the use of the high-cost SOI wafer.

In order to avoid the process compatibility and the stress problem associated with the boron-doping dissolving wafer process, we have developed a double-sided dry-etching micromachining process on a regular silicon wafer to form microprobes as the substrate for the ultrasound transducer array. Different from the SOI micromachined probes, this silicon 


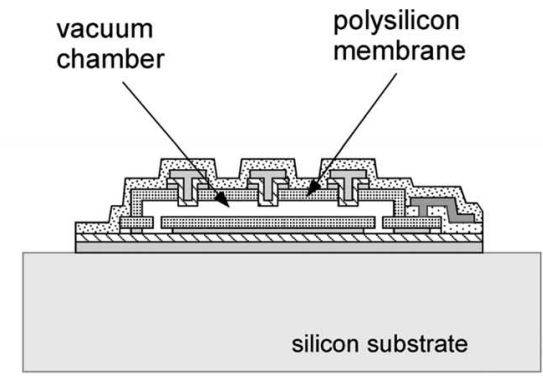

(a)

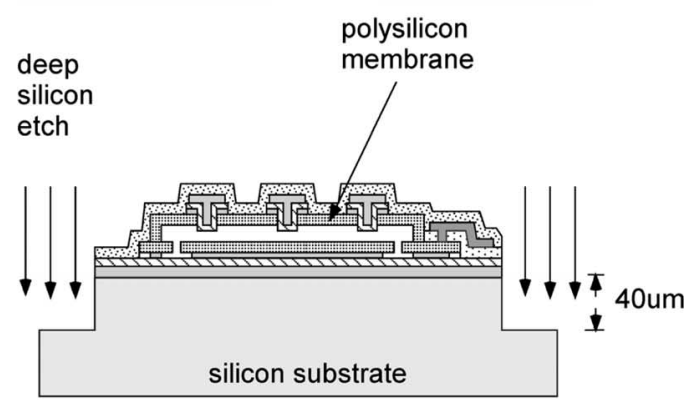

(b)

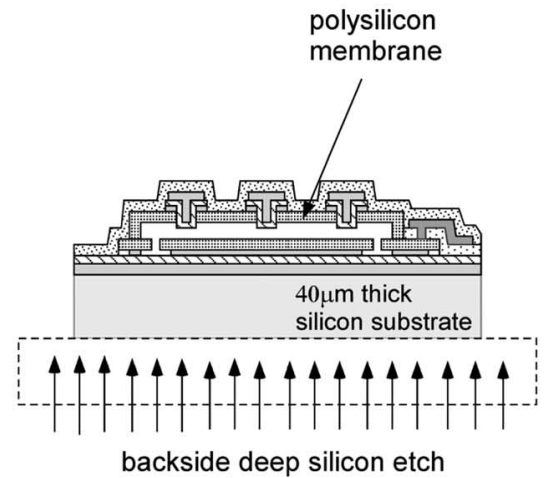

(c)

Fig. 4. Double-sided dry etching used to micromachine the substrate of the CMUT array.

probe uses regular silicon wafers as the substrate and has a cost advantage. Similar to the SOI silicon probes, the dimensions of this double-side dry-etched probe can be controlled within $\pm 2 \mu \mathrm{m}$. We use traditional thin-film surface micromachining process to build the ultrasound transducers and the metal electrical interconnect on a silicon substrate, as shown in Fig. 4(a). After the completion of these thin-film structures, a $40-\mu \mathrm{m}$-wide $40-\mu \mathrm{m}$-deep trench is formed on the frontside around the peripheral of the array using deep silicon etching, as shown in Fig. 4(b), and a flood deep silicon etching from the backside is used to release the devices, as shown in Fig. 4(c). To form an integrated dielectric ribbon cable, an additional backside photolithography is needed to create a recess under the ribbon cable area such that after the flood backside etching, the silicon substrate under the ribbon cable is completely etched away.

In addition to miniaturization, one of the advantages of micromachining the substrate thickness is the reduction of crosstalk from Lamb waves propagating in the substrate. This is important to improve the signal-to-noise ratio of the transducer.
The crosstalk between neighboring CMUT devices is partly caused by the Lamb wave, which refers to the elastic modes of ultrasound propagation in a solid plate with free boundaries. When Lamb wave modes propagate in a fluid loaded silicon wafer, they suffer leakage by mode conversion into the fluid. Due to the dispersive nature of Lamb waves, the radiation pattern of the Lamb waves in a silicon wafer is a function of the plate thickness. As the wafer is made thicker, the Lamb wave leaks more energy into water due to the change of its velocity that corresponds to the change of its critical angle. According to this result, a thinner wafer is more desirable for crosstalk reduction. From [17], the Lamb wave-related crosstalk level is reduced by $2.3 \mathrm{~dB}$ if the substrate thickness is reduced from 550 to $40 \mu \mathrm{m}$, as used in this device design.

\section{Microfabrication Process}

The fabrication of this device started with a polysilicon surface micromachining process, which is similar to the traditional polysilicon multiuser MEMS processes (MUMPs, Cronos Integrated Microsystems, Research Triangle Park, NC, USA) to form the CMUT array, and it was finished by a two-sided deep silicon etching to micromachine the silicon substrate into a probe shape. The fabrication of this MEMS ultrasonic transducer array started with a p-type 4-in (100) silicon wafer. The surface of the wafer was first doped with phosphorus by using a diffusion process at $950{ }^{\circ} \mathrm{C}$ for $60 \mathrm{~min}$ to form a highly conductive surface area. This area was used to reduce charge feed-through for the operation of electrostatic devices to be built on the wafer. A layer of silicon dioxide, which is approximately $1.6 \mu \mathrm{m}$ thick, was next grown thermally, followed by a deposition of $5000 \AA$ of silicon nitride and $250 \AA$ of silicon dioxide using LPCVD. The purpose of the top thin silicon dioxide is to separate the silicon nitride film from the polysilicon microstructure. A polysilicon of $5000 \AA$ was then deposited by using LPCVD and doped with phosphorus using a diffusion process at $950{ }^{\circ} \mathrm{C}$ for $30 \mathrm{~min}$. The sheet resistance of the polysilicon film was 10-12 $\Omega$ /square after the diffusion and the postannealing. The polysilicon film was patterned by using a photolithography process and a reactive ion etching to form electrodes, electrical interconnects, and anchoring pads for the polysilicon structures to be built in the subsequent step. A thin layer of PSG, which is typically $0.1-0.2 \mu \mathrm{m}$ thick after annealing, was next deposited by using LPCVD. The sacrificial oxide was patterned by using a photolithography process and reactive ion etching to form anchoring holes. A layer of polysilicon film, which is typically $1.0-2.5 \mu \mathrm{m}$ thick, was next deposited by using LPCVD and doped with phosphorus, using a diffusion process at $950{ }^{\circ} \mathrm{C}$. The polysilicon film was annealed in nitrogen gas at $1100^{\circ} \mathrm{C}$ for $60 \mathrm{~min}$ for stress relief. The stress of the structural polysilicon film was between -5 and $-20 \mathrm{MPa}$ after the annealing process. A photolithography process and a reactive ion etching were used to pattern the structural polysilicon. This step defined the shape of the polysilicon membranes while simultaneously carving an array of $2 \mu \mathrm{m} \times 2 \mu \mathrm{m}$ holes in the polysilicon membrane. The holes in the membrane will harbor an array of dielectric posts in the polysilicon membrane as a mechanical stop. Without these dielectric posts, 


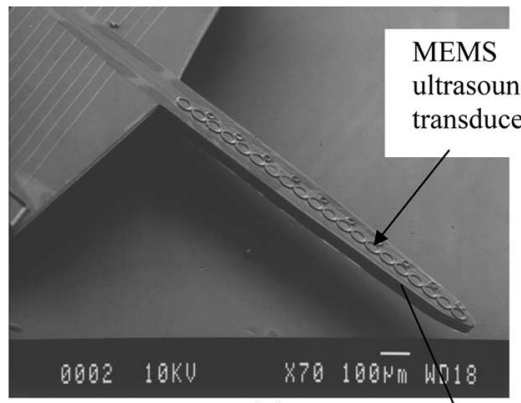

(a)

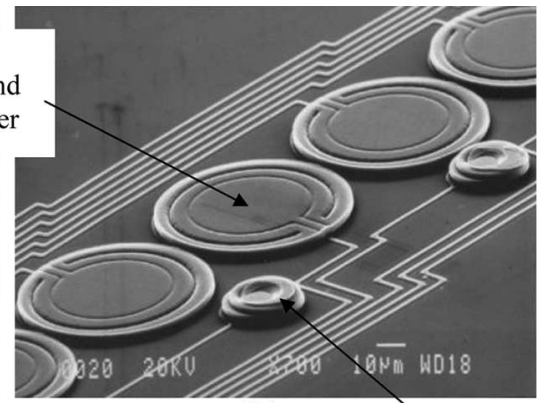

(b)

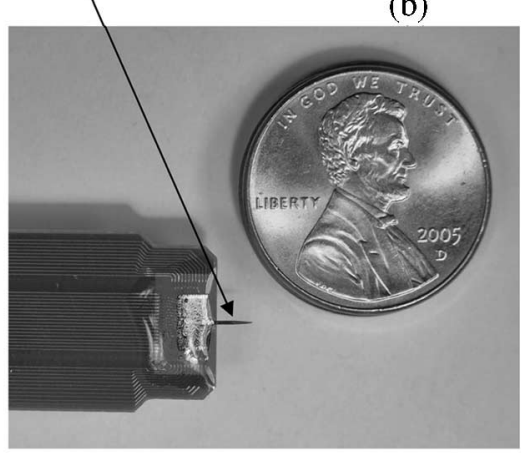

Iridium

electrode

(c)

Fig. 5. (a) SEM photograph of a 1-D CMUT array. (b) Close-up of the ultrasonic transducer element. (c) Photograph of the probe-shaped CMUT array after being bonded on a printed circuit board.

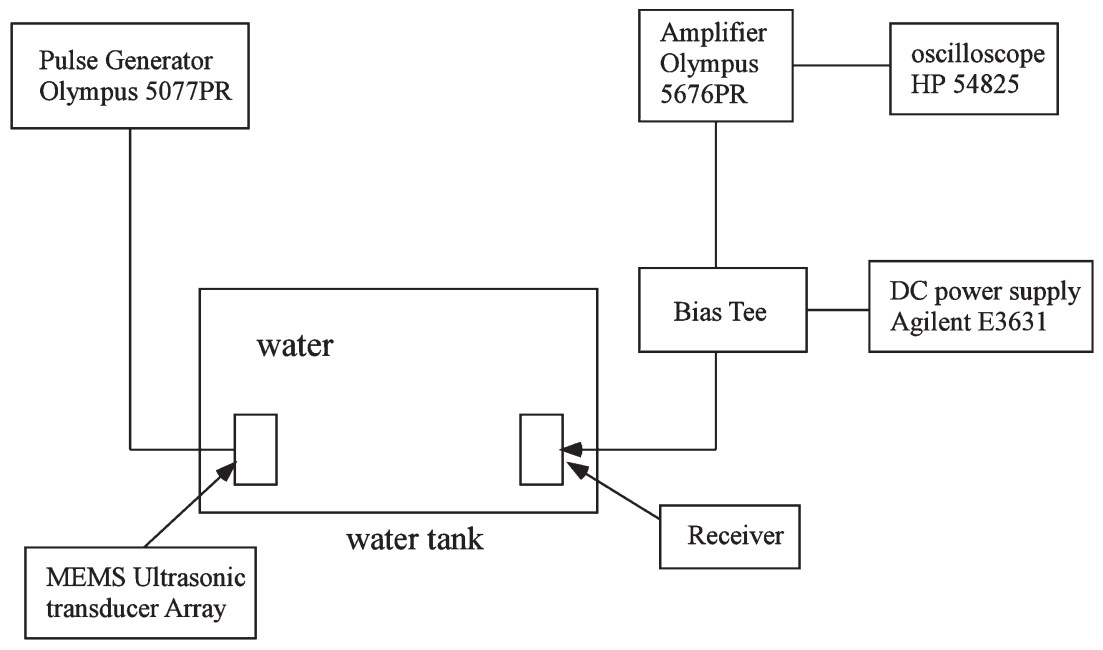

Fig. 6. Setup for transmission and reception experiment.

the polysilicon membrane could short to the counter electrode during transducer operation. The dry etching used to pattern the structural polysilicon was intentionally overetched into the sacrificial oxide $250-400 \AA$ deep. The dielectric post molded by these pits will therefore protrude the same distance down from the lower surface of the polysilicon membrane. A thermal oxidation was then used to convert the surface of the polysilicon membrane into a thin $(\sim 250 \AA)$ layer of silicon dioxide. Next, $2000 \AA$ of silicon nitride was deposited by using LPCVD. This layer and the thin thermal oxide underneath were patterned by using the same mask, forming an array of dielectric posts in the polysilicon membrane, as shown in Fig. 3. The wafer was next etched in $49 \% \mathrm{HF}$ for removing the sacrificial oxide, freeing the membrane structures. A super critical drying process was used after the release etching to prevent the stiction problem.
The sacrificial release process slightly undercuts the thin silicon oxide layer under the polysilicon microstructures. It also undercuts the thin thermal oxide sandwiched between the top nitride layer and the polysilicon membrane around the dielectric posts. Due to the very thin $(\sim 250 \AA)$ oxide used in this sandwiched structure, which limits the diffusion of the etchant into/out from a very narrow gap, these undercuts were insignificant and did not degrade the anchor structures. The wafer was then coated with $3 \mu \mathrm{m}$ of PECVD tetraethoxysilane (TEOS). This TEOS layer was used to seal the release holes, so that the chamber under the membrane was kept at vacuum. A photolithography process and a wet etching were used to pattern this TEOS layer, leaving only TEOS around the release holes. Next, a $0.3-\mu \mathrm{m}$ thick layer of silicon dioxide was deposited by using PECVD. This layer was used to passivate the polysilicon membrane and 
separated the metal interconnects from the silicon nitride. This PECVD oxide layer was patterned by using a photolithography process and a reactive ion etching, which opened via holes on the metal-polysilicon connection area. After another photolithography process defined the metal-layer pattern, $4000 \AA$ of aluminum was sputtered and patterned by using a lift-off process. A total of $1.1 \mu \mathrm{m}$ of parylene $\mathrm{C}$ is coated by using thermal evaporation and then patterned. This patterning process exposes metal on the intended bonding pad area.

After completion of the surface micromachining processes, a photolithography process was used to define a 20 - $\mu \mathrm{m}$-wide window around the peripheral of each CMUT array. The dielectric layers in this window were completely removed by using a reactive ion etching. A deep silicon etching using surface technology system (Imperial Park, Newport, U.K.) etching system was next used to create a $40-\mu$ m-deep trench on the window opening. The wafer was flipped and glued to a carrying silicon wafer and then etched from the backside using a dry deep silicon etching process until the trenches on the frontside were exposed. This etching process released each individual device from the substrate. The devices were separated from the carrying silicon substrate by a wet etching using acetone.

\section{Device Characteristics}

Fig. 5(a) shows the SEM photograph of a 1-D ultrasonic transducer array. The probe-shaped silicon substrate is $40 \mu \mathrm{m}$ thick, $80 \mu \mathrm{m}$ wide, and $2.5 \mathrm{~mm}$ long. Fig. 5(b) shows the close-up of the ultrasonic transducers and the nearby iridium electrodes. These electrodes are designed for electrical recording or stimulation from/to cells for simultaneous application of ultrasound to the cells. Fig. 5(c) shows a photograph of the probe-shaped CMUT array after being bonded on a printed circuit board. Ultrasonic transducers with drum diameter ranging from 20 to $90 \mu \mathrm{m}$ were fabricated and tested. Four different gap heights (the distance between the drum membrane and its counter electrode) used for theses devices are 0.10, 0.12, 0.16, and 0.20 $\mu \mathrm{m}$. UMECH MEMS motion analyzer G2 was used to measure the frequency and amplitude response of the membrane in air under electrical excitation at different frequencies and voltages. From these measurements, we found that there is a discrepancy of up to $11 \%$ between the experimental and simulation results about the diaphragm displacement under dc bias. In numerical simulation, we assume the membrane anchors to the substrate around the whole peripheral of the membrane. In real device, the anchor was divided into multiple sections by release holes. This impacts the accuracy of the simulation. The device was then tested in a water tank. Fig. 6 shows the setup for acoustic measurement. A pulser generator (Olympus 5077PR, Center Valley, PA, USA) transmits a short pulse, which is typically $20-500 \mathrm{~ns}$ wide with a peak-to-peak amplitude of 20-100 V, to drive the transducer. The ultrasounds generated by the transducer are detected either by a hydrophone (piezoceramic needle hydrophone TNU100A, NTR, Seattle, WA, USA) or a CMUT array of the same design. The output of the hydrophone or the MEMS ultrasonic transducer array was amplified by $40 \mathrm{~dB}$ by a high-gain bandpass amplifier (Olympus 5676PR, Center

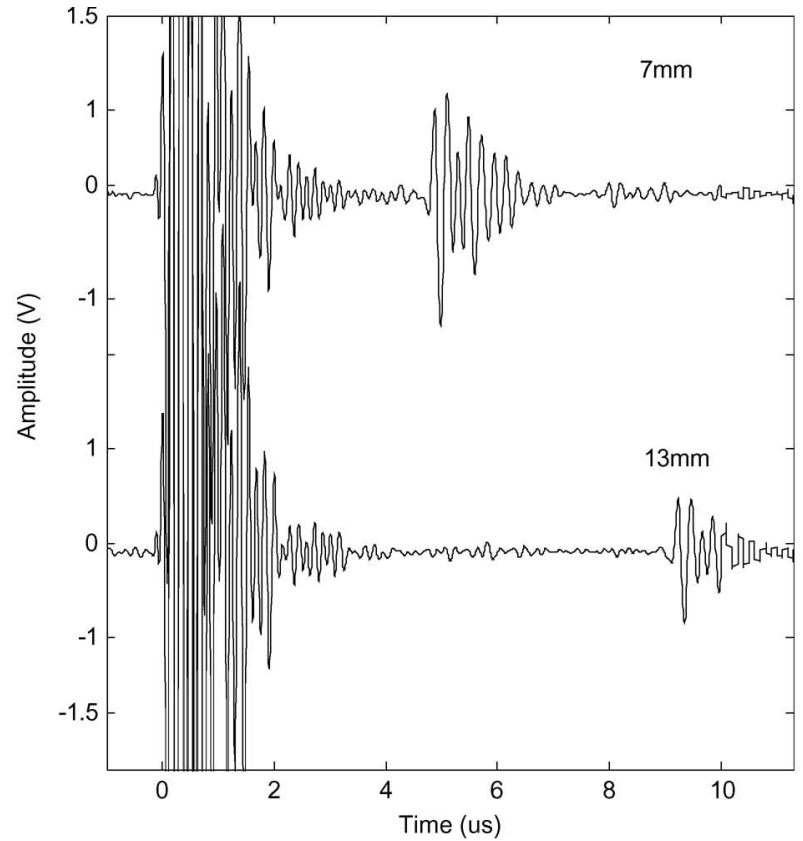

(a)

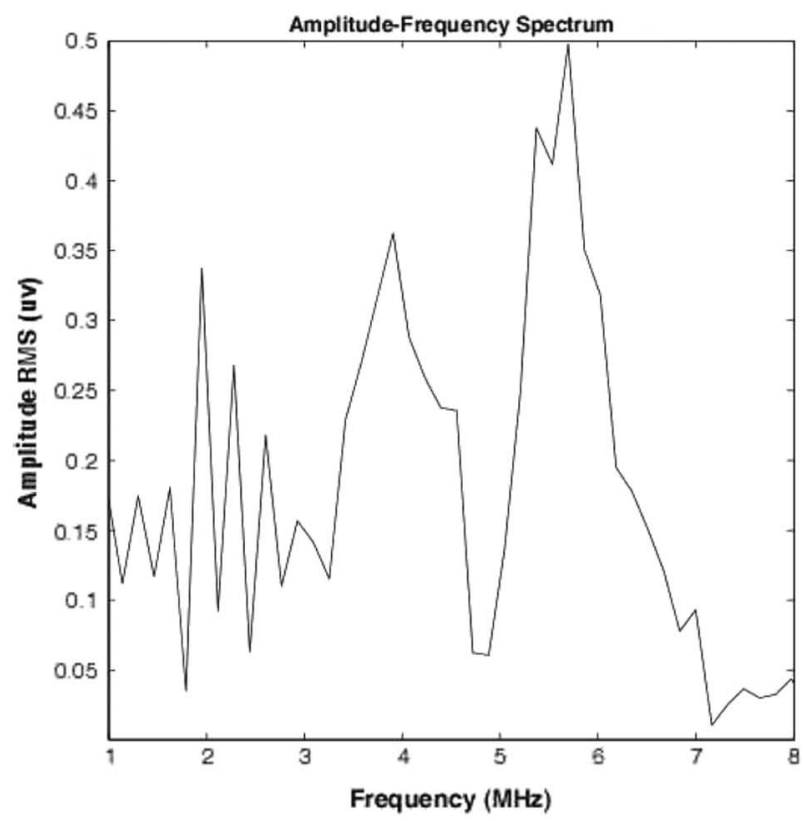

(b)

Fig. 7. (a) Ultrasound signals generated by a $46-\mu$ m-diameter transducer by applying a single electrical pulse on transducer membrane. The upper figure was measured $7 \mathrm{~mm}$, whereas the lower figure was measured $13 \mathrm{~mm}$ from the source transducer. (b) Frequency response of a 46- $\mu$ m-diameter transducer. The major resonant frequency is $5.9 \mathrm{MHz}$.

Valley, PA, USA) before being digitized by an oscilloscope (HP 54825 Oscilloscope, Palo Alto, CA, USA). To improve the signal-to-noise ratio, signals were averaged 64 times. Fig. 7 shows the ultrasound signals generated by a $46-\mu \mathrm{m}$-diameter transducer by applying an 80 -ns-wide $40-\mathrm{V}$ peak-to-peak single electrical pulse without dc bias on the transducer membrane. The polysilicon membrane thickness of this device was $1.0 \mu \mathrm{m}$, whereas its gap height between the membrane and the counter electrode was $0.18 \mu \mathrm{m}$. The upper figure of Fig. 7(a) shows 


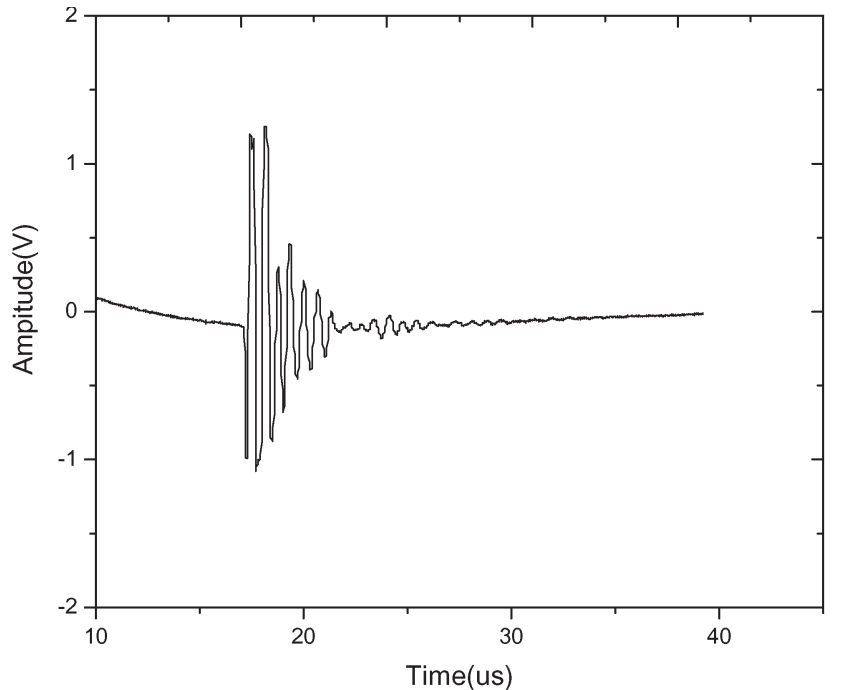

(a)

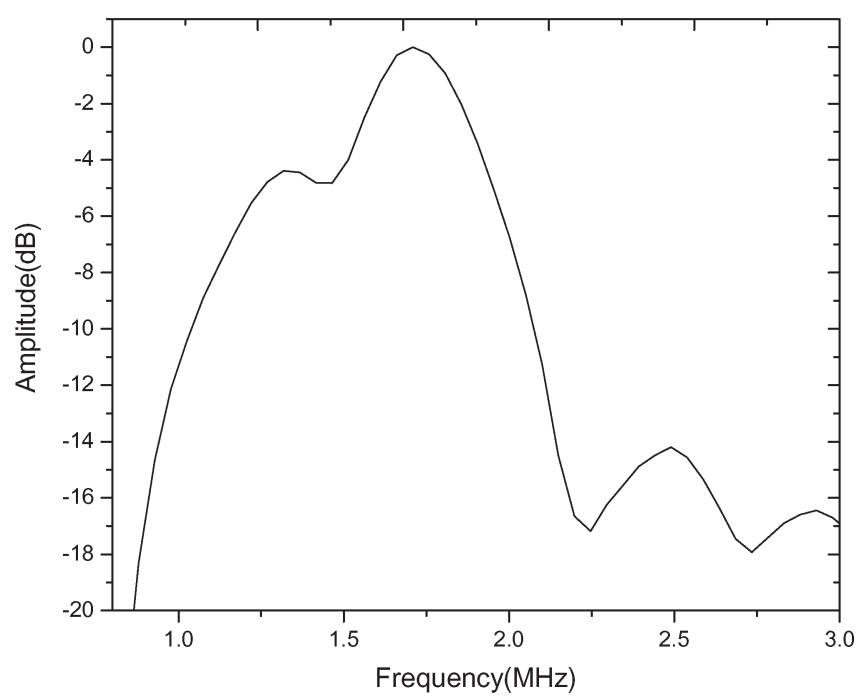

(b)

Fig. 8. Ultrasonic signal and its frequency response transmitted by an $80-\mu \mathrm{m}-$ diameter device when triggered by a 500 -ns-wide $100-\mathrm{V}$ peak-to-peak excitation without dc bias. The ultrasonic signal was received by a commercial hydrophone.

the ultrasound signal measured $7 \mathrm{~mm}$, whereas the lower figure measured $13 \mathrm{~mm}$ from the source CMUT array by a hydrophone. Fig. 7(b) shows the frequency response. The fundamental resonant frequency is $5.9 \mathrm{MHz}$.

Fig. 8 shows the ultrasonic signal transmitted by a $80-\mu \mathrm{m}-$ diameter device when it was triggered by a single 500-ns-wide $100-\mathrm{V}$ peak-to-peak voltage without dc bias. The polysilicon membrane thickness of this device was $1.0 \mu \mathrm{m}$, whereas its gap height was $0.17 \mu \mathrm{m}$. The ultrasonic signal recorded in Fig. 8 was received by a hydrophone (piezoceramic hydrophone PZT-Z44-0400, SEA, Sunnyvale, CA, USA) which was placed $2.2 \mathrm{~cm}$ from the CMUT array. The central frequency of this acoustic transducer was $1.6 \mathrm{MHz}$, whereas its $-6-\mathrm{dB}$ fractional bandwidth was $50 \%$. Fig. 9 shows the ultrasonic signal and its spectrum, which are transmitted by the same $80-\mu \mathrm{m}$-diameter device while received by a CMUT array of the same design as the transmitter. The receiver CMUT array was biased by a $10-\mathrm{V}$

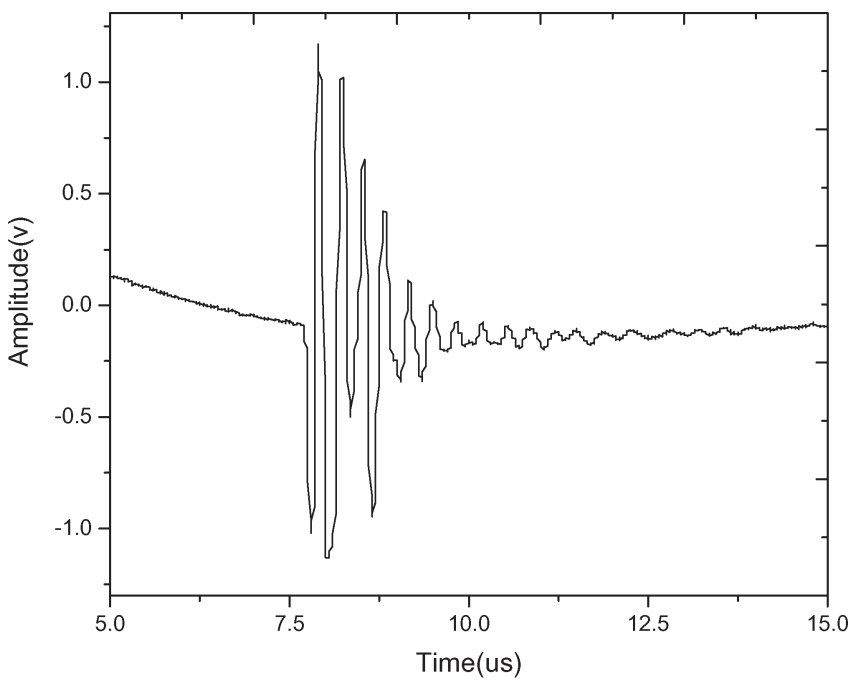

(a)

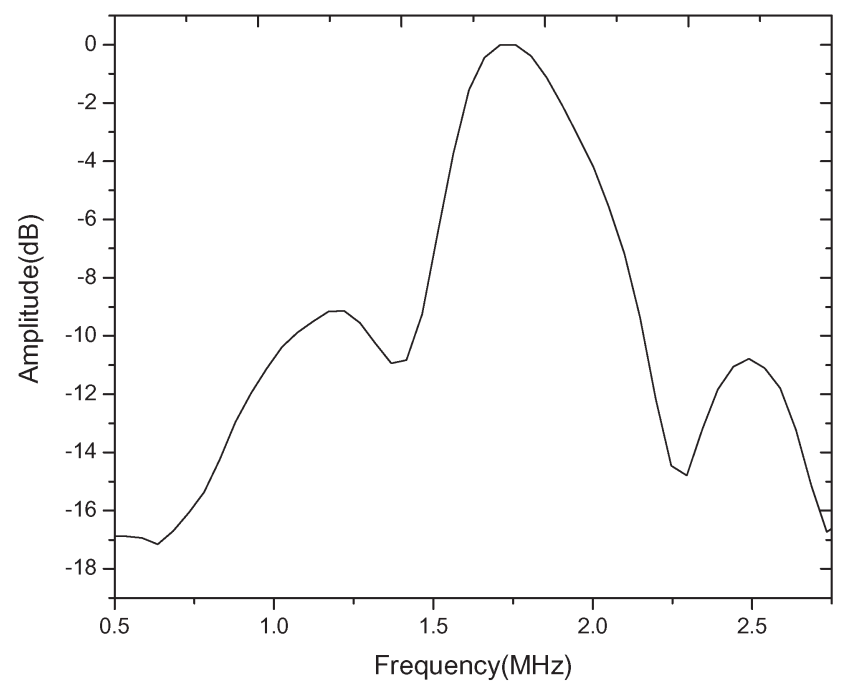

(b)

Fig. 9. Ultrasonic signal and its frequency response transmitted by an $80-\mu \mathrm{m}-$ diameter device when triggered by a 500 -ns-wide $100-\mathrm{V}$ peak-to-peak excitation without dc bias. The ultrasonic signal was received by another CMUT array of the same design. The receiver transducer was biased with $10-\mathrm{V}$ dc bias.

dc bias. The central frequency of the spectrum was $1.8 \mathrm{MHz}$, whereas the $-6-\mathrm{dB}$ fractional bandwidth was $34 \%$.

\section{IMAGING CHARACTERISTIC}

One of the applications of this transducer array is ultrasonic imaging. The array has been used to shoot images of a metal wire phantom in water. The experimental setup for image formation is shown in Fig. 10. A section of metal wire was soaked in water as the target to be imaged. Instead of a motor positioning system, a manual positioning system is used to avoid electromagnetic interference. A 1-D probe-shaped CMUT array and a commercial 1-MHz ultrasound transducer (Panametrics V302, Waltham, MA, USA) are fixed on two ends of an L-shape holder. The pulser/receiver (Panametrics PR5072, Waltham, MA, USA) transmits a short pulse to drive the piezoelectric transducer. Then, the reflected wave is received by the CMUT array. The echoes are amplified by $59 \mathrm{~dB}$ 


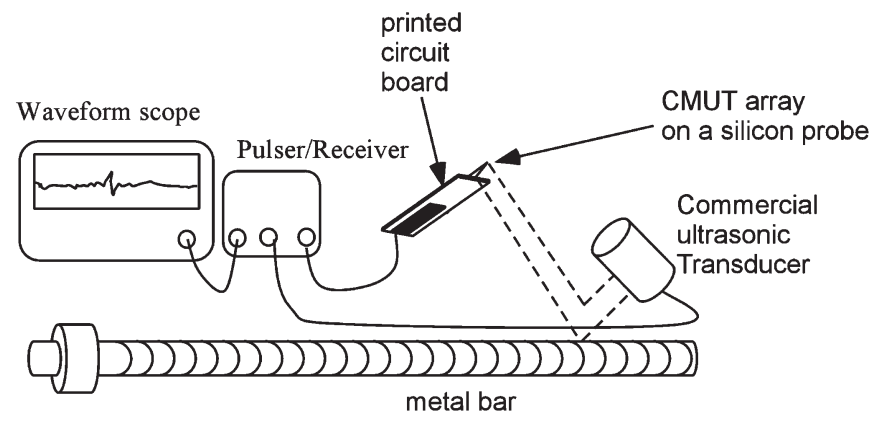

Fig. 10. Setup for image acquisition of a metal wire using a probe-shaped CMUT array.

using the same pulser/receiver before being digitized by an oscilloscope (Tektronix TDS 3054B, Tokyo, Japan). To improve the signal-to-noise ratio, signal averaging of 1024 times at each holder is performed before the holder is moved to the next step position. The step size between two scan positions is $100 \mu \mathrm{m}$. Signal processing is performed and displayed on a computer using MATLAB (MathWorks, Natick, MA, USA). Fig. 11(a) and (b) shows the ultrasonic signals received by an array of 56- $\mu$ m-diameter CMUT imagers and the frequency response. The polysilicon membrane thickness and the gap height of the receiver CMUT are 1.0 and $0.17 \mu \mathrm{m}$, respectively. Fig. 11(c) shows the B-mode image of a metal wire shot by this CMUT array. The right-hand side bar shows the brightness contrast scale of the image.

\section{Ultrasound DOPPLER Flow MEASUREMENT}

Ultrasonic Doppler blood flowmeter is widely used in clinic and medical research. Ultrasound waves change frequency as they bounce off the red blood cells moving in the blood. Changes in frequency can be used to measure both the direction and the speed of blood flow. Many commercial Doppler ultrasonic systems use either one ultrasonic transducer element or a small array to measure the blood flow. Due to the wavelengthrelated penetration depth limit, these traditional Doppler ultrasonic systems generally have to compromise between flow sensitivity and penetration depth. For example, the commercial Doppler ultrasonic system is incapable of monitoring the blood flow distribution in cortex due to the slower flowing speed (about $1 \mathrm{~mm} / \mathrm{s}$ ). In neuroscience and pharmacology research, the blood flow in cortex is an important parameter for evaluating the function state of the brain. The probe-shaped CMUT array described in this paper provides a unique tool for blood flow rate monitoring. Since the distance between the neighboring transducer elements in this array is small $(3 \mu \mathrm{m})$ and accurately defined by the microfabrication process, the Doppler frequency shift for blood flow measurement can be accurately identified.

Fig. 12 shows the working principle of blood flow measurement using this transducer array. Assume that the array is facing a blood vessel in the same plane. One of the transducers (labeled 0) is working as the source transducer to emit ultrasounds. The transmitted ultrasonic wave will hit the blood flowing in the vessel and bounce back. The echo will be

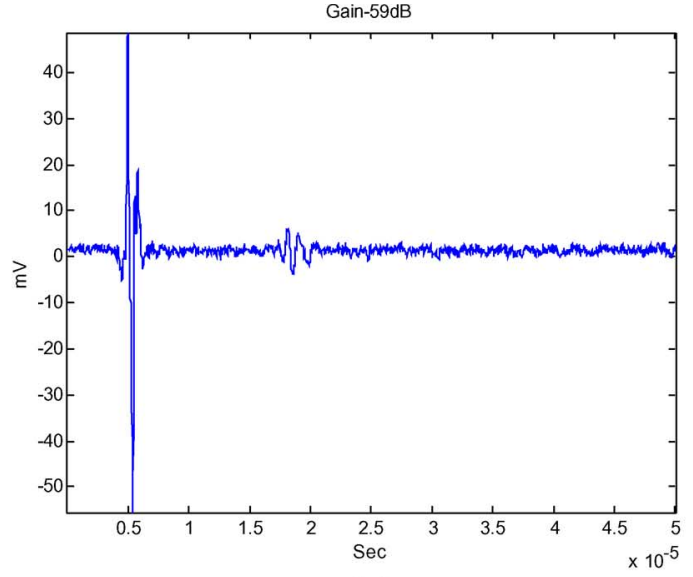

(a)

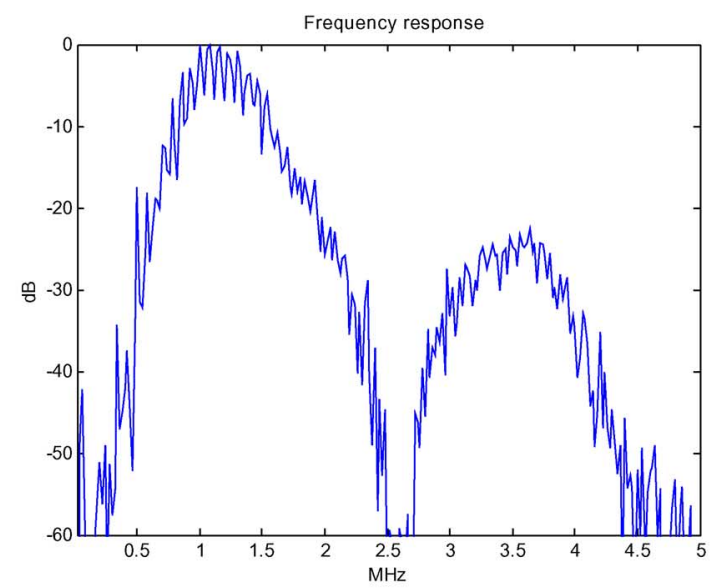

(b)

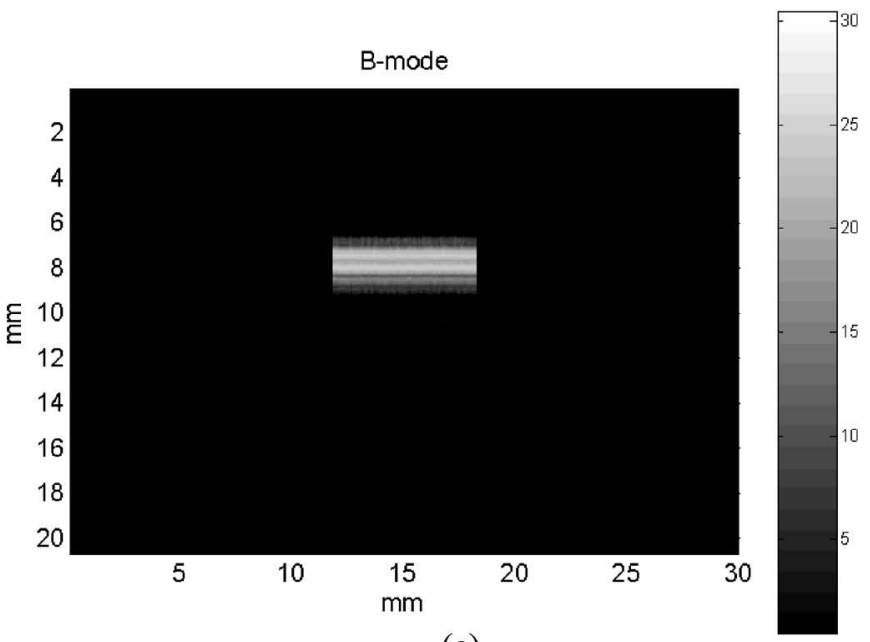

(c)

Fig. 11. (a) Ultrasonic signals received by an array of $56-\mu$ m-diameter imagers. (b) Frequency response of the received signals. (c) B-mode image of a metal wire

detected by all the transducers in the array. The echo frequency detected by transducer $i$ can be expressed by

$$
f_{i}=\frac{C+V_{\mathrm{o}}}{C-V_{i}} f_{\mathrm{o}}
$$

where $C$ is the speed of ultrasound in the tissue, $V_{\mathrm{o}}$ is the blood flow velocity projected on transducer 0 , and $V_{i}$ is the blood flow velocity projected on transducer $i$. 


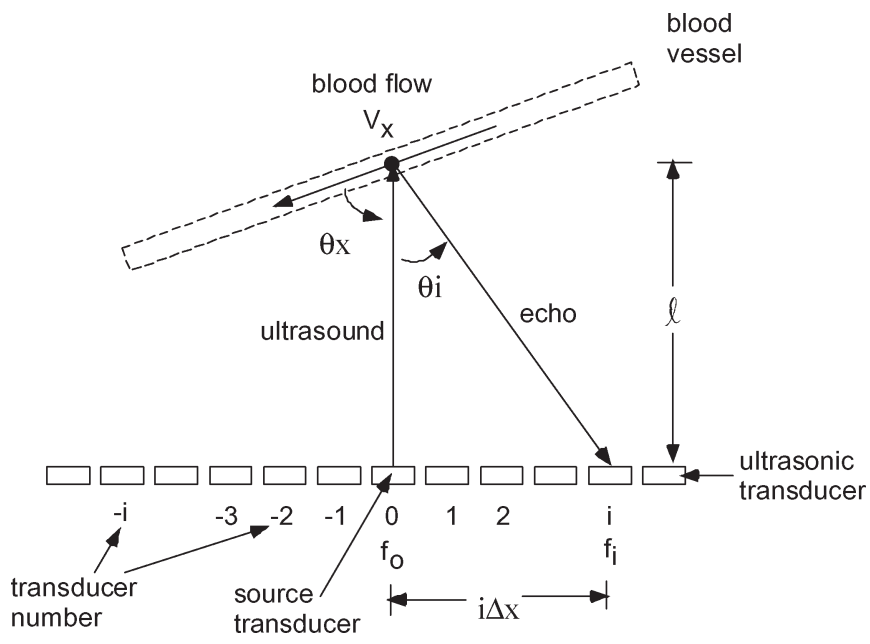

Fig. 12. Application of this CMUT array as an ultrasonic Doppler blood flowmeter. The pitch of the ultrasonic phase array is defined by photolithography and the dry etching used in the microfabrication of this device. This array makes it possible to accurately relate the phase shift of the echoes to the flow rate. Arrays comprising 24-32 ultrasonic transducers were designed for this application

The projected blood flow velocity $V_{i}$ observed by transducer $i$ is given by

$$
V_{i}=V_{x} \cos \left(\theta_{x}+\theta_{i}\right)
$$

where $\theta_{x}$ is the angle between the blood flow and the travel direction of impinging ultrasound wave (emitted by transducer $0), \theta_{i}$ is the angle between the echo toward the $i$ th sensor and the impinging ultrasound wave from transducer $0, V_{x}$ is the blood flow rate, $f_{\mathrm{o}}$ is the frequency of the original ultrasound wave emitted by transducer 0 , and $f_{i}$ is the frequency of echo ultrasounds detected by transducer $i$.

In this ultrasonic transducer array, the pitch is defined by design and the microfabrication process and is accurately controlled. As a result, the transducer-to-transducer distance is known, and $\theta_{i}$ for any transducer in the array can be determined by

$$
\theta_{i}=\tan ^{-1}\left(\frac{i \Delta x}{l}\right)
$$

where $\Delta x$ is the pitch of the transducer array, and $l$ is the distance from the source transducer (here is sensor 0 ) to the blood vessel. The two unknowns, namely, $\theta_{x}$ and $\mathrm{V}_{x}$, can therefore be determined by solving (3) and (4). In fact, for a 24-element ultrasound array, (3) and (4) will provide 24 linear equations for determining the blood flow vector $\left(\theta_{x}, V_{x}\right)$. This is the case of overdetermined linear questions, and the leastsquare solution for the vector can be solved.

\section{A. Preliminary Flow Measurement}

A board-level front-end circuit and a data processing unit were developed to analyze the ultrasound signals recorded from the probe-shaped 1- and 2-D $(2 \times 16)$ CMUT arrays (with 24-32 transducer elements) in the Doppler flow measurement. A roller pump was used to circulate a blood mimicking fluid (CIRS blood mimicking fluid model 046, Norfolk, VA, USA) through a rubber tube with an inner diameter of $4.8 \mathrm{~mm}$. The rubber tube was soaked in a water tank. The CMUT array was mounted in the water tank and oriented $60^{\circ}$ with respect to the orientation of the flow tube as the transmitter. In this preliminary experiment, both a CMUT array and a commercial hydrophone (piezoceramic hydrophone PZT-Z44-0400, SEA, Sunnyvale, CA, USA) were used as the receiver. The Doppler shift recorded from the receiving transducers was demodulated by using the software approach. In order to get an optimal resolution of the Doppler frequency shift, an autoregressive model (AR) [19], [20] was used for data analysis. Matlab simulation was used to determine the optimal AR model order. A 40-dB preamplifier with an equivalent input noise of $1.4 \mathrm{nV} /$ $\sqrt{\mathrm{Hz}}$ and a $40-\mathrm{dB}$ passband amplifier were cascaded to amplify the received ultrasound signal. A 12-b AD converter was used to provide digital data for demodulation. A laser Doppler flowmeter (Moor Instruments Laser Doppler Monitor DRT4, Devon, U.K.) was used in a parallel measurement for calibration purposes. By using the pulse wave Doppler mode with a pulse repetition ratio of $10 \mathrm{kHz}$, a burst frequency of $2 \mathrm{MHz}$, a burst count of ten, and a burst amplitude of $40 \mathrm{~V}$, a flow rate resolution of $1 \mathrm{~cm} / \mathrm{s}$ per $10 \mathrm{~Hz}$ of Doppler frequency shift was observed. A discrepancy of $6.5 \%$ was observed between the flow rate measured from the CMUT array and the laser Doppler flowmeter.

\section{CONCLUSion}

An ultrasonic transducer array for minimally invasive medical diagnosis has been developed. Integrated on a silicon probe smaller than a human hair, this transducer array is able to reach deep inside the tissue with a minimum tissue disruption to emit and receive ultrasounds. Adept at close target placement, this device can use high-frequency ultrasounds to interact with tissue or organs. It overcomes the resolution limit of traditional noninvasive ultrasound transducers in exploring detailed biological information deep inside the tissue/organ. Ultrasonic transducers with a polysilicon membrane diameter from 20 to $90 \mu \mathrm{m}$ and a thickness from 1.0 to $2.5 \mu \mathrm{m}$ have been designed and prototyped. These transducers are capable of emitting and sensing ultrasounds from 1 to $10 \mathrm{MHz}$. Any 1- or 2-D array with a reasonable transducer count can be integrated on this micromachined probe substrate. By integrating an array of drum membranes of different geometrical dimensions, a broad frequency spectrum can be generated and detected from the devices on the same substrate built by the same fabrication process. This device is useful for small area, high-precision ultrasound diagnosis, or stimulation, for example, near-field imaging of cells or tissue, measurement of blood flow rate in fine vessels, and stimulation of cell membrane to alter the cell membrane permeability.

\section{REFERENCES}

[1] T. I. Weng, H. P. Wang, W. J. Chen, L. B. Chin, and L. M. Ng, "Ultrasound diagnosis of occult femoral hernia presenting with intestinal obstruction," Amer. J. Emerg. Med., vol. 19, no. 4, pp. 333-334, 2001.

[2] L. A. Frizzell, P. J. Benkeser, K. B. Ocheltree, and C. A. Cain, "Ultrasound phased array for hyperthermia treatment," in Proc. IEEE Ultrason. Symp., 1985, pp. 930-935. 
[3] J. C. Kaimal and J. E. Gaynor, "Another look at sonic thermometry," Boundary-Layer Meteorol., vol. 56, no. 4, pp. 401-410, Sep. 1991.

[4] C. Z. Rosen, B. V. Hiremath, and R. E. Newnham, Piezoelectricity. New York: AIP, Apr. 1992

[5] A. Arnau and A. Arnau Vives, Piezoelectric Transducers and Applications. Berlin, Germany: Springer-Verlag, May 2004.

[6] C. L. de Korte, G. Pasterkamp, A. F. van der Steen, H. A. Woutman, and N. Bom, "Characterization of plaque components with intravascular ultrasound elastography in human femoral and coronary arteries in vitro," Circulation, vol. 102, no. 6, pp. 617-623, Aug. 2000.

[7] R. Carlton and A. McKenna Adler, "Fundamentals of medical imaging," in Principles of Radiographic Imaging: An Art and a Science, 3rd ed. Florence, KY: Thomson Delmar Learning, Sep. 2000.

[8] J. Knight and F. Degertekin, "Capacitive micromachined ultrasonic transducers for forward looking intravascular imaging arrays," in Proc. IEEE Ultrason. Symp., 2002, pp. 1079-1082.

[9] Y. Huang, A. S. Ergun, M. H. Badi, and B. T. Khuri-Yakub, "Fabricating capacitive micromachined ultrasonic transducers with wafer-bonding technology," J. Microelectromech. Syst., vol. 12, no. 2, pp. 128-137, Apr. 2003.

[10] A. Bozkurt, I. Ladabaum, A. Atalar, and B. T. Khuri-Yakub, "Theory and analysis of electrode size optimization for capacitive microfabricated ultrasonic transducers," IEEE Trans. Ultrason., Ferroelectr., Freq. Control, vol. 46, no. 6, pp. 1364-1374, Nov. 1999.

[11] Z. H. Cho et al., Foundations of Medical Imaging. New York: WileyInterscience, 1993.

[12] D. J. Anderson, K. Najafi, S. J. Tanghe, D. A. Evans, K. L. Levy, J. F. Hetke, X. Xue, J. J. Zappia, and K. D. Wise, "Batch fabricated thin-film electrodes for stimulation of the central auditory system," IEEE Trans. Biomed. Eng., vol. 36, no. 7, pp. 693-704, Jul. 1989.

[13] P. Norlin et al., "A 32-site neural recording probe fabricated by DRIE of SOI substrates," J. Micromech. Microeng., vol. 12, no. 4, pp. 414-419, Jun. 2002.

[14] S. J. Tanghe, K. Najafi, and K. D. Wise, "A planar IrO multichannel stimulating electrode for use in neural prosthesis," Sens. Actuators, vol. B1, pp. 464-467, Jan. 1990.

[15] T. B. Avallone, III and E. A. Avallone, Mark's Standard Handbook for Mechanical Engineers, 10th ed. New York: McGraw-Hill, 1996.

[16] J. Chen, K. D. Wise, J. F. Hetke, and S. C. Bledsoe, Jr., "A multichannel neural probe for selective chemical delivery at the cellular level," IEEE Trans. Biomed. Eng., vol. 44, no. 8, pp. 760-769, Aug. 1997.

[17] Y. Roh, Y. Kim, and K. Kang, "Finite element analysis on reduction of the cross talk in ultrasonic transducers," in Proc. SPIE Smart Struct. Mater., vol. 4693, pp. 214-221.

[18] G. Korosoglou, S. E. Hrdt, R. Bekeredjian, J. Jenne, M. Konstantin, M. Hagemueller, H. A. Katus, and H. Kuecherer, "Ultrasound exposure can increase the membrane permeability of human neutrophil granulocytes containing microbubbles without causing complete cell destruction," Ultrasound Med. Biol., vol. 32, no. 2, pp. 297-303, Feb. 2006.

[19] A. Herment, G. Demoment, and J. F. Gioviannelli, "Adaptive estimation of spectrum and mean frequency of Doppler signals," in Proc. IEEE Ultrason. Symp., 1994, pp. 1717-1720.

[20] P. I. J. Keeton, F. S. Schlindwein, and D. H. Evans, "A study of the spectral broadening of simulated Doppler signals using FFT and AR modelling," Ultrasound Med. Biol., vol. 23, no. 7, pp. 1033-1045, 1997.

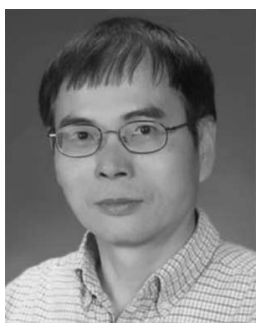

Jingkuang Chen received the B.S. and M.S. degrees in electrical engineering from the National Taiwan University, Taipei, Taiwan, R.O.C., and the Ph.D. degree in electrical engineering from the University of Michigan, Ann Arbor, in 1996. His Ph.D. dissertation focused on the development of microfluidic systems on silicon for selective drug delivery to the central nervous system and for inkjet printing.

From 1996 to 2004, he was a Member of Research Staff with Xerox Wilson Research Center, Webster, NY, working on the development of SOI MEMS optical systems and plastic microfluidic devices for printing application. In 2004, he joined the faculty of the University of New Mexico, Albuquerque, where he is currently an Associate Professor with the Department of Electrical and Computer Engineering. His research interests are invasive capacitive micromachined ultrasonic transducer arrays that incorporate ultrasound with light sources and/or a drug-delivery network on a miniature platform for imaging, diagnosis, and treatment.

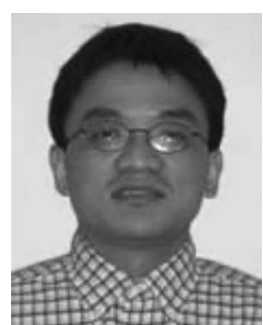

Xiaoyang Cheng received the B.S. degree in mechanical engineering from Huazhong University of Science and Technology, Wuhan, China, and the M.S. degree in electromechanical engineering from the South China University of Technology, Guangzhou, China. He is currently working toward the Ph.D. degree in electrical engineering in the Department of Electrical and Computer Engineering, University of New Mexico, Albuquerque.

His research focuses on the design, fabrication, and characterization of invasive MEMS capacitive micromachined ultrasonic transducers for biomedical applications.

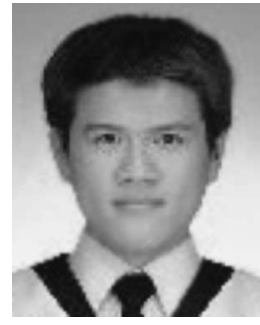

Chien-Chang Chen was born in Taiwan, R.O.C. He received the B.S. degree in physics from the National Central University, Jhongli, Taiwan, in 2003, and the M.S. degree in electrical engineering from the National Chiao Tung University, Hsinchu, Taiwan, in 2006. His thesis was entitled, "A closed-form integral model of spiral inductor using the Kramers-Kronig relations."

$\mathrm{He}$ is currently working on solid-state theory for RF device modeling.

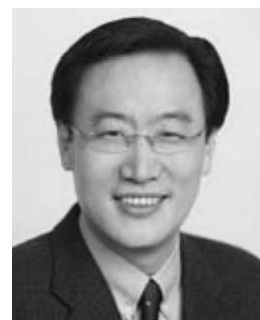

Pai-Chi Li (S'93-M'95-SM'01) received the B.S degree in electrical engineering from the National Taiwan University, Taipei, Taiwan, R.O.C., in 1987, and the M.S. and Ph.D. degrees from the University of Michigan, Ann Arbor, in 1990 and 1994, respectively, both in electrical engineering systems

From 1990 to 1994, he was a Research Assistant with the Department of Electrical Engineering and Computer Science, University of Michigan. In June 1994, he joined Acuson Corporation, Mountain View, CA, as a Member of Technical Staff. His work at Acuson was primarily in the areas of medical ultrasonic imaging system design for both cardiology and general imaging applications. In August 1997 he again joined the Department of Electrical Engineering, National Taiwan University, where he is currently a Professor with the Department of Electrical Engineering and the Director of the Institute of Biomedical Electronics and Bioinformatics. He is on the Editorial Board of Ultrasonic Imaging. His current research interests include biomedical ultrasonic imaging and signal processing.

Dr. $\mathrm{Li}$ is an Associate Editor for the IEEE TRANSACTIONS ON Ultrasonics, FERROElECTRICS, AND FREQUENCY CONTROL. He is the recipient of the 2002 Outstanding Young Electrical Engineer Award and the 2005 Outstanding Electrical Engineering Professor Award from the Chinese Institute of Electrical Engineering, the 2002 Dr. Wu Dayou Research Award and the 2004 Distinguished Research Award from the National Science Council, the 2003 Outstanding Researcher Award and the 2004 Distinguished Research Achievement Award from the National Taiwan University, and the Distinguished Industrial Collaboration Award from the Ministry of Education. $\mathrm{He}$ also received the Distinguished Achievement Award in Electrical Engineering: Systems in 1994 for his outstanding academic achievement at the University of Michigan. 


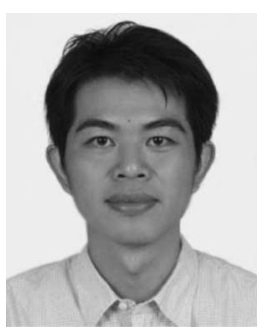

Jian-Hung Liu received the B.S. degree in electrical engineering from the Southern Taiwan University of Technology, Tainan, Taiwan, R.O.C., in 2002, and the M.S. degree in bioengineering from the National Yang Ming University, Taipei, Taiwan, in 2004. He is currently a Ph.D. candidate in electrical engineering in the Department of Electrical Engineering, National Taiwan University, Taipei.

His current research interests include highfrequency small-animal imaging and high-frequency array transducers.

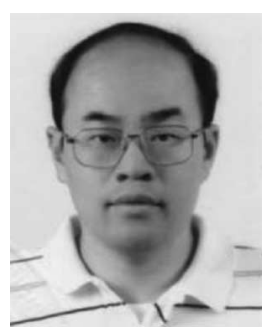

Yu-Ting Cheng was born in Taiwan, R.O.C. He received the B.S. and M.S. degrees in materials science and engineering from the National Tsing-Hua University, Hsinchu, Taiwan, in 1991 and 1993, respectively, the M.S. degree in materials science and engineering from Carnegie Mellon University, Pittsburgh, PA, in 1996, and the Ph.D. degree in electrical engineering from the University of Michigan, Ann Arbor, in 2000. His Ph.D. dissertation involved the development of novel vacuum packaging technique for MEMS applications.

He has two years of army service in Taiwan. After graduation, he joined the IBM Thomas J. Watson Research Center, Yorktown Heights, NY, as a Research Staff Member and was involved in several system-on-package (SoP) projects. In 2002, he joined the National Chiao Tung University, Hsinchu, as an Assistant Professor in the Department of Electronics Engineering, where he is currently an Associate Professor. His research interests include the fundamental study of materials for microsystems integration and MEMS applications, SoP, and the design and fabrication of microsensors and microactuators.

Dr. Cheng is a member of the Institute of Physics and Phi Tau Phi. 